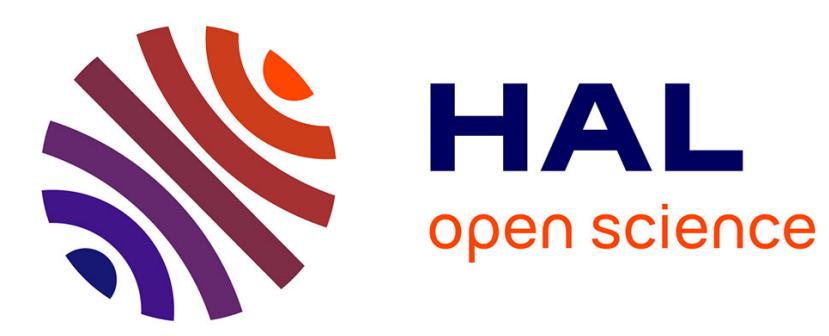

\title{
Persistence and propagation in periodic reaction-diffusion models
}

Francois Hamel, Lionel Roques

\section{To cite this version:}

Francois Hamel, Lionel Roques. Persistence and propagation in periodic reaction-diffusion models. Tamkang Jourmal of Mathematics, 2014, 45, pp.217-228. hal-01257404

\author{
HAL Id: hal-01257404 \\ https://hal.science/hal-01257404
}

Submitted on 18 Jan 2016

HAL is a multi-disciplinary open access archive for the deposit and dissemination of scientific research documents, whether they are published or not. The documents may come from teaching and research institutions in France or abroad, or from public or private research centers.
L'archive ouverte pluridisciplinaire HAL, est destinée au dépôt et à la diffusion de documents scientifiques de niveau recherche, publiés ou non, émanant des établissements d'enseignement et de recherche français ou étrangers, des laboratoires publics ou privés. 


\title{
Persistence and propagation in periodic reaction-diffusion models
}

\author{
François Hamel ${ }^{a}$ and Lionel Roques ${ }^{b *}$ \\ a Aix Marseille Université, CNRS, Centrale Marseille \\ Institut de Mathématiques de Marseille, UMR 7373, 13453 Marseille, France \\ \& Institut Universitaire de France \\ b INRA, UR546 Biostatistique et Processus Spatiaux, 84914 Avignon, France
}

\begin{abstract}
In this paper, we consider Fisher-KPP reaction-diffusion models in periodic environments. We review some results on the questions of species persistence and propagation of pulsating traveling waves. We study the role of the heterogeneities and the fragmentation of the environment on the persistence and on the propagation speeds.
\end{abstract}

\section{Introduction}

This paper is concerned with the study of the qualitative properties and propagation phenomena for solutions of reaction-diffusion equations of the type

$$
\frac{\partial u}{\partial t}-\nabla \cdot(D(x) \nabla u)=f(x, u), \quad t>0, x \in \mathbb{R}^{N}
$$

in the whole space $\mathbb{R}^{N}$ with periodic coefficients. Such equations arise in various population dynamics models in biology, ecology and genetics, see e.g. [9, 33, 44]. The unknown function $u$ stands for the density of a species and is always assumed to be nonnegative.

Throughout the paper, the matrix field $D: \mathbb{R}^{N} \rightarrow M^{N \times N}(\mathbb{R})$ is assumed to be symmetric, of class $C^{1, \alpha}\left(\mathbb{R}^{N}\right)$ with $\alpha>0$, and uniformly elliptic in the sense that

$$
\exists \nu>0, \quad \forall x \in \mathbb{R}^{N}, \quad \forall \xi \in \mathbb{R}^{N}, \quad \sum_{1 \leq i, j \leq N} D_{i, j}(x) \xi_{i} \xi_{j} \geq \nu \sum_{1 \leq i \leq N} \xi_{i}^{2} .
$$

${ }^{*}$ This work has been carried out in the framework of the Labex Archimède (ANR-11-LABX-0033) and of the A*MIDEX project (ANR-11-IDEX-0001-02), funded by the "Investissements d'Avenir" French Government program managed by the French National Research Agency (ANR). The research leading to these results has received funding from the European Research Council under the European Union's Seventh Framework Programme (FP/2007-2013) / ERC Grant Agreement n.321186 - ReaDi - Reaction-Diffusion Equations, Propagation and Modelling. 
The term $\nabla \cdot(D(x) \nabla u)$ corresponds to a diffusion term, which may be anisotropic. The reaction term $f: \mathbb{R}^{N} \times[0,+\infty) \rightarrow \mathbb{R}$ is assumed to be of class $C^{0, \alpha}$ with respect to $x \in \mathbb{R}^{N}$ locally uniformly in $u \in[0,+\infty)$, and of class $C^{1}$ with respect to $u \in[0,+\infty)$. Moreover, we assume that the state 0 is a steady state, that large constants are supersolutions and that the per capita growth rate is decreasing with respect to the density $u$, in the sense that

$$
\left\{\begin{array}{l}
f(x, 0)=0 \text { for all } x \in \mathbb{R}^{N}, \\
\exists M>0, \quad f(x, u) \leq 0 \text { for all } x \in \mathbb{R}^{N} \text { and } u \geq M
\end{array}\right.
$$

and

$$
u \mapsto \frac{f(x, u)}{u} \text { is decreasing in }(0,+\infty) \text { for all } x \in \mathbb{R}^{N} .
$$

We focus in this paper on the influence of periodic heterogeneities on the properties of the solutions $u$ of (1.1). Namely, the functions $D$ and $f(\cdot, u)$ (for every $u \geq 0$ ), are assumed to be $L$-periodic with respect to $x$ in the sense that there exists $L=\left(L_{1}, \cdots, L_{N}\right) \in(0,+\infty)^{N}$ such that

$$
D\left(\cdot+L_{i} e_{i}\right)=D \text { and } f\left(\cdot+L_{i} e_{i}, u\right)=f(\cdot, u) \text { in } \mathbb{R}^{N}
$$

for all $1 \leq i \leq N$, where $\left(e_{i}\right)_{1 \leq i \leq N}$ denotes the canonical basis of $\mathbb{R}^{N}$.

The particular case $N=1, D(x)$ constant and $f(x, u)=u(1-u)$ correspond to the logistic model of Fisher [16] and Kolmogorov, Petrovsky and Piskunov [28]. Shigesada, Kawasaki and Teramoto [44,45] have considered the particular case $f(x, u)=u(r(x)-\gamma(x) u)$ where $r$ and $\gamma$ are $C^{0, \alpha}\left(\mathbb{R}^{N}\right)$ periodic functions with $\min _{\mathbb{R}^{N}} \gamma>0$.

\section{Persistence}

In this section, we study the asymptotic behavior at large time of the solutions of (1.1) with bounded nonnegative initial conditions $u_{0}$ which are not identically equal to zero. These conditions on $u_{0}$ are assumed throughout the paper. It follows then from the maximum principle and the assumptions on $f$ that, given such a $u_{0}$, there exists a unique, classical, solution $u$ in $(0,+\infty) \times \mathbb{R}^{N}$, with $0<u(t, x) \leq \max \left(M,\left\|u_{0}\right\|_{L^{\infty}\left(\mathbb{R}^{N}\right)}\right)$ for all $t>0$ and $x \in \mathbb{R}^{N}$. We are interested in the asymptotic persistence or extinction of the population, in the following sense. We say that a solution $u$ of (1.1) tends to extinction if $u(t, x) \rightarrow 0$ as $t \rightarrow+\infty$ uniformly in $x \in \mathbb{R}^{N}$, and we say that $u$ is asymptotically persistent if $\liminf _{t \rightarrow+\infty}\|u(t, \cdot)\|_{L^{\infty}\left(\mathbb{R}^{N}\right)}>0$. Actually, it will turn out (see Theorem 2.1 below) that, under the assumptions of Section 1, $u$ is asymptotically persistent if and only if it does not tend to extinction, that is if and only if $\lim \sup _{t \rightarrow+\infty}\|u(t, \cdot)\|_{L^{\infty}\left(\mathbb{R}^{N}\right)}>0$. In the case of persistence, an important question is to determine the limit of $u(t, x)$, if any, as $t \rightarrow+\infty$. We will in particular focus on the influence of the heterogeneities of the medium, that is the diffusion and reaction coefficients $D(x), f(x, \cdot)$ and the period $L$, on the extinction or persistence.

\subsection{An analytic criterion for the persistence}

The extinction or the persistence of the population turns out to depend strongly on the stability of the steady state 0 . The question of the stability of 0 is expressed in terms of 
the principal eigenvalue of the linearized equation around the state 0 . Namely, it follows from Krein-Rutman theory that there is a unique real number $\lambda[D, r]$, called the principal eigenvalue, for which there exists a (unique up to multiplication) function $\phi \in C^{2, \alpha}\left(\mathbb{R}^{N}\right)$, called principal eigenfunction, solving

$$
\left\{\begin{array}{l}
-\nabla \cdot(D(x) \nabla \phi)-r(x) \phi=\lambda[D, r] \phi \text { in } \mathbb{R}^{N} \\
\phi \text { is } L \text {-periodic, } \phi>0 \text { in } \mathbb{R}^{N}
\end{array}\right.
$$

with $r(x)=\frac{\partial f}{\partial u}(x, 0)$ denotes the derivative of $f$ with respect to $u$ at $u=0$. Notice in particular that, if $r$ is equal to a constant $r_{0}$, then $\phi$ is constant and $\lambda\left[D, r_{0}\right]=-r_{0}$. It turns out that the extinction vs. persistence only depends on the sign of $\lambda[D, r]$, as the following result shows:

Theorem 2.1 [6] Assume that the diffusion matrix D satisfies (1.2) and that the function $f$ satisfies (1.3) and (1.4).

(i) (Persistence) If $\lambda[D, r]<0$, then there exists a unique, classical, solution $p$ of the stationary problem

$$
\nabla \cdot(D(x) \nabla p)+f(x, p(x))=0 \text { in } \mathbb{R}^{N}, \quad p>0 \text { in } \mathbb{R}^{N}
$$

and this solution is actually L-periodic. Furthermore, the solutions $u(t, x)$ of (1.1) converge to $p(x)$ as $t \rightarrow+\infty$ locally uniformly in $\mathbb{R}^{N}$.

(ii) (Extinction) If $\lambda[D, r] \geq 0$, then 0 is the only nonnegative stationary solution of (1.1) and any solution $u(t, x)$ of (1.1) converges to 0 as $t \rightarrow+\infty$ uniformly in $x \in \mathbb{R}^{N}$.

This result gives a necessary and sufficient condition for the persistence, that is the negativity of the principal eigenvalue $\lambda[D, r]$. Roughly speaking, if the state 0 (where the species is absent) is unstable (in the sense that $\lambda[D, r]<0$ ), then the solution $u$ cannot converge to 0 at large time, and the converse also holds, whatever the (bounded) initial condition may be. Furthermore, in the case of persistence, all solutions $u$ of (1.1) converge locally uniformly in $x \in \mathbb{R}^{N}$ as $t \rightarrow+\infty$ to the same limiting state $p(x)$, which turns out to periodic and separated from 0 . Actually, one of the difficult points in the proof of the uniqueness of $p$ is to show, by unsing some estimates on the principal eigenvalues of the linearized operator in large balls with Dirichlet boundary conditions, that, if $\lambda[D, r]<0$, any steady state $p$ of $(2.2)$ is actually bounded from below by a positive constant (this point is not present in the proof of similar results in bounded domains with various boundary conditions $[1,9]$ ). This remarkably simple criterion for persistence vs. extinction involves the spatial features of the environment and the biological features of the species, but it is important to notice that the growth rate only appears through its per capita limit $r(x)$ at $u=0$. Lastly, we mention that similar results as in Theorem 2.1 have been obtained with formal arguments in periodic media with piecewise constant coefficients [44]. 


\subsection{Effect of the heterogeneity and the fragmentation of the medium}

In this section, we use the criterion mentioned in Theorem 2.1 to study the role of the spatial structure of the environment on species persistence. For the sake of simplicity, we assume here that the diffusion matrix $D$ is constant and equal to the identity matrix $D=I$ and we therefore only focus on the role of the intrinsic growth rate coefficient $r$. We set $\lambda[r]=\lambda[I, r]$ and we also point out that the variational formulation

$$
\lambda[r]=\min _{\phi \in H_{l o c}^{1}\left(\mathbb{R}^{N}\right) \backslash\{0\}, \phi \text { is } L \text {-periodic }} \frac{\int_{C}|\nabla \phi|^{2}-r \phi^{2}}{\int_{C} \phi^{2}}
$$

holds as well for $L^{\infty}\left(\mathbb{R}^{N}\right) L$-periodic functions $r$, where $C=\left[0, L_{1}\right] \times \cdots \times\left[0, L_{N}\right]$ denotes the periodicity cell.

Let us first compare an arbitrary medium with a homogeneous one where the growth rate coefficient has the same average.

Theorem 2.2 There holds $\lambda[r] \leq \lambda[\bar{r}]=-\bar{r}$, where $\bar{r}$ denotes the average of $r$ :

$$
\bar{r}=\frac{1}{L_{1} \times \cdots \times L_{N}} \int_{C} r(x) d x .
$$

The proof is immediate, since on the one hand $\lambda[r] \leq-\bar{r}$ by taking $\phi=1$ in (2.3) and, on the other hand, $\lambda[\bar{r}]=-\bar{r}$ as already noticed before Theorem 2.1. Despite its simplicity, Theorem 2.2 has an interesting meaning: a heterogeneous environment gives better chance for persistence than a homogeneous one with the same average. Furthermore, the chances for persistence are all the higher as the repartition of the resources is unbalanced, in the following sense.

Theorem 2.3 [6] If $\bar{r} \geq 0$ and $r$ is not identically equal to 0 , then $\lambda[$ Ar] $<0$ for all $A>0$, the $\operatorname{map}[0,+\infty) \ni A \mapsto \lambda[A r]$ is decreasing and $\lambda[A r] \rightarrow-\infty$ as $A \rightarrow+\infty$.

As an application, consider the case $r=\bar{r}+A r_{1}$, where $r_{1}$ has zero average and is not constant. One has $\lambda[r]=\lambda\left[\bar{r}+A r_{1}\right]=-\bar{r}+\lambda\left[A r_{1}\right]$. If $\bar{r}<0$ and $A=0$ (homogeneous medium with a negative intrinsic growth rate), then $\lambda[r]=-\bar{r}>0$ and the population tends to extinction. But Theorem 2.3 implies that $\lambda\left[A r_{1}\right] \rightarrow-\infty$ as $A \rightarrow+\infty$, whence $\lambda[r]=\lambda\left[\bar{r}+A r_{1}\right]=-\bar{r}+\lambda\left[A r_{1}\right] \rightarrow-\infty$ as $A \rightarrow+\infty$. In other words, if the heterogeneities have a large enough amplitude, then the population persists, even though the medium is unfavorable in the average sense.

Remark 2.4 When $\bar{r}<0$, the map $[0,+\infty) \ni A \mapsto \lambda[A r]$ may or may not be monotone, according to the profile of $r$. For instance, if $r$ is equal to a negative constant $r=r_{0}<0$, then $\lambda[A r]=-A r_{0}$ is increasing with respect to $A$. Consider now the case where $\bar{r}<0$ and $\max _{\mathbb{R}^{N}} r>0$. Then there exist $\delta>0$ and a non-empty ball $B \subset C$ such that $r \geq$ 
$\delta>0$ in $B$, whence $\lambda[A r] \leq \lambda_{D}(-\Delta-A r, B) \leq \lambda_{D}(-\Delta, B)-A \delta \rightarrow-\infty$ as $A \rightarrow+\infty$, where $\lambda_{D}(-\Delta-A r, B)$ and $\lambda_{D}(-\Delta, B)$ denote respectively the principal eigenvalues of the operators $-\Delta-A r$ and $-\Delta$ in $B$ with Dirichlet boundary conditions on $\partial B$. But $\lambda[0]=0$ (with constant eigenfunctions) and $\lambda[A r] / A \rightarrow-\bar{r}>0$ as $A \rightarrow 0^{+}$, after integration of (2.1) with $D=I$ and $A r$ instead of $r$. Therefore, the map $A \mapsto \lambda[A r]$ is not monotone on $[0,+\infty)$ if $\bar{r}<0$ and $\max _{\mathbb{R}^{N}} r>0$. Notice also that the map $A \mapsto \lambda[A r]$ is always concave, due to the variational formula $(2.3)$.

Let us now study the influence of the location of the heterogeneities of the medium, when they have a given average and even a given distribution. More precisely, for any $L$-periodic function $r \in L^{\infty}\left(\mathbb{R}^{N}\right)$, we denote $\mu_{r}: \mathbb{R} \rightarrow[0,+\infty)$ the distribution function of $r$, defined by

$$
\mu_{r}(t)=|\{x \in C, r(x)>t\}|
$$

where $|E|$ denotes the Lebesgue measure of a measurable set $E \subset \mathbb{R}^{N}$. If two functions $r$ and $\widetilde{r}$ have the same distribution functions $\mu_{r}=\mu_{\tilde{r}}$, then they have the same average and equimeasurable superlevel sets, but the locations of these sets may be different. The goal of the last part of this section is to see that if the resources are arranged in a special way, then the chances for species persistence are better. More precisely, given any measurable bounded $L$ periodic function $r$, the periodic symmetric decreasing Steiner rearrangement of $r$ with respect to the variable $x_{i}$ (with $1 \leq i \leq N$ ) is the unique $L$-periodic measurable bounded function $r^{* i}$ which is symmetric with respect to the hyperplane $\left\{x_{i}=L_{i} / 2\right\}$, nonincreasing with respect to $x_{i}$ for $L_{i} / 2 \leq x_{i} \leq L_{i}$ and such that, for almost every $\left(x_{1}, \ldots, x_{i-1}, x_{i+1}, \ldots, x_{N}\right) \in \mathbb{R}^{N-1}$ and for every $t \in \mathbb{R}$, the sets $\left\{x_{i} \in\left[0, L_{i}\right], r(x)>t\right\}$ and $\left\{x_{i} \in\left[0, L_{i}\right], r^{* i}(x)>t\right\}$ have the same measure.

Theorem $2.5[6]$ Under the above notations, one has $\lambda\left[r^{* i}\right] \leq \lambda[r]$.

The proof of this result mainly follows from the variational formula (2.3) and from standard Hardy-Littlewood and Polya-Szegö rearrangement inequalities. By performing several consecutive Steiner rearrangements with respect to any variables, the principal eigenvalue always decreases, in the sense that

$$
\lambda\left[r^{* i, * j}\right] \leq \lambda\left[r^{* i}\right] \leq \lambda[r]
$$

Notice that, in general, $r^{* i, * j} \neq r^{* j, * i}$ and $\lambda\left[r^{* i, * j}\right] \neq \lambda\left[r^{* j, * i}\right]$.

As a particular example of these general properties, consider the case where the environment is binary in the sense that $r$ takes only two values. More precisely, let us assume here that there exists a measurable set $C^{+}=C^{+}(r) \subset C$ and two constants $r_{-}<r_{+}$such that

$$
\left\{\begin{array}{l}
r(x)=r^{+} \text {if } x \in C^{+}, \\
r(x)=r^{-} \text {if } x \in C^{-}=C \backslash C^{+},
\end{array}\right.
$$

and $r$ is $L$-periodic. The set $C^{+}$corresponds to the favorable regions and the set $C^{-}$to the unfavorable ones. The distribution function of such a binary function $r$ depends only on $r^{ \pm}$and 
on the proportion $h=\left|C^{+}\right| /|C|$ of favorable environment. For given $r^{-}<r^{+}$and $h \in(0,1)$, it is a natural question to look for an optimal habitat configuration, that is a binary $L$-periodic function $r$ taking values $r^{ \pm}$, having the given proportion $h$ of favorable environment and minimizing the principal eigenvalue $\lambda[r]$ in this class. In dimension $N=1$, the answer is immediate and the optimal configuration is the one for which, up to shifts, $C^{+}$is an interval of size $h L_{1}$ (included in $\left[0, L_{1}\right]$ ). The simple looking problem in higher dimensions $N \geq 2$ is actually quite intricate and it is still open. However, it is possible to prove that there exist optimal configurations. Furthermore, up to shifts in space, these optimal configurations are equal to their Steiner symmetrizations with respect to all variables $x_{i}$ and the favorable region is connected and convex in all variables $x_{i}$. Lastly, the optimal shapes for the optimal favorable and unfavorable regions $C^{ \pm}$strongly depend on the amplitude $r^{+}-r^{-}$of the heterogeneities of the growth rate $r$, and they are not always balls or slabs even if they look like balls or slabs: we refer to [40] for further results in this direction and for enlightening numerical simulations.

Remark 2.6 For a diffusion matrix $D$ and a function $f$ satisfying the general assumptions of Section 1, with $r=\frac{\partial f}{\partial u}(\cdot, 0)$, the formula (2.3) is replaced by

$$
\lambda[D, r]=\min _{\phi \in H_{l o c}^{1}\left(\mathbb{R}^{N}\right) \backslash\{0\}, \phi \text { is } L \text {-periodic }} \frac{\int_{C} D \nabla \phi \cdot \nabla \phi-r \phi^{2}}{\int_{C} \phi^{2}} .
$$

In particular, the map $B \mapsto \lambda[B D, r]$ is nondecreasing on $(0,+\infty)$ and, for every $B>0$, there holds $-\max _{\mathbb{R}^{N}} r \leq \lambda[B D, r] \leq-\bar{r}$ (by choosing $\phi=1$ for the upper inequality). It is also easy to see that $\lambda[B D, r] \rightarrow-\bar{r}$ as $B \rightarrow+\infty$. Indeed, consider any sequence $\left(B_{n}\right)_{n \in \mathbb{N}}$ of positive real numbers converging to $+\infty$ and let $\phi_{n}$ be the principal eigenfunctions of the operators $-\nabla \cdot\left(B_{n} D \nabla\right)-r$ with periodicity conditions, that is

$$
-\nabla \cdot\left(B_{n} D(x) \nabla \phi_{n}\right)-r(x) \phi_{n}=\lambda\left[B_{n} D, r\right] \phi_{n} \text { in } \mathbb{R}^{N}
$$

with $\phi_{n}>0$ in $\mathbb{R}^{N}$ and $\phi_{n}$ is $L$-periodic. Up to normalization, one can assume without loss of generality that $\left\|\phi_{n}\right\|_{L^{2}(C)}=\sqrt{|C|}$ for all $n \in \mathbb{N}$. By multiplying (2.4) by $\phi_{n}$ and integrating over $C$, it follows that $B_{n} \int_{C} D(x) \nabla \phi_{n} \cdot \nabla \phi_{n}-\int_{C} r(x) \phi_{n}^{2}=\lambda\left[B_{n} D, r\right]|C|$. Since the sequences $\left(\lambda\left[B_{n} D, r\right]\right)_{n \in \mathbb{N}}$ and $\left(\left\|\phi_{n}\right\|_{L^{2}(C)}\right)_{n \in \mathbb{N}}$ are bounded and since $B_{n} \rightarrow+\infty$, one infers from (1.2) that $\left\|\nabla \phi_{n}\right\|_{L^{2}(C)} \rightarrow 0$ as $n \rightarrow+\infty$, whence $\phi_{n} \rightarrow 1$ as $n \rightarrow+\infty$ in $L^{2}(C)$ (and thus in $L^{1}(C)$ ) by Poincaré-Wirtinger inequality. Integrating (2.4) over $C$ and passing to the limit as $n \rightarrow+\infty$ implies that $\lambda\left[B_{n} D, r\right] \rightarrow-\bar{r}$ as $n \rightarrow+\infty$.

\section{Propagation}

In this section, we go back to the reaction-diffusion equation (1.1) with periodic diffusion and reaction coefficients $D$ and $f$ satisfying (1.2), (1.3) and (1.4), and we assume that $\lambda[D, r]<0$. In other words, from Theorem 2.1, there is a unique positive $L$-periodic solution $p$ of $(2.2)$ and the solutions $u(t, x)$ of (1.1) converge to $p(x)$ locally uniformly in $x \in \mathbb{R}^{N}$ as $t \rightarrow+\infty$. We focus here on how and at which speed the steady state $p(x)$ invades the unstable steady state 0 . We will study the influence of the heterogeneities and the fragmentation of the medium on the propagation speeds of the pulsating fronts connecting 0 and $p$. 


\subsection{Pulsating fronts}

For a homogeneous equation of the type $u_{t}=D_{0} u_{x x}+f(u)$ with $D_{0}>0$ being a positive constant and $f$ satisfying (1.3) and (1.4), a traveling front (moving to the right) is a solution of the type $u(t, x)=\phi(x-c t)$ with $\phi(-\infty)=M_{0}>\phi>0=\phi(+\infty)$, where $M_{0}$ denotes the unique positive zero of $f$. In the moving frame with speed $c$ to the right, a traveling front $u(t, x)=\phi(x-c t)$ is invariant in time. Such fronts are known to exist if and only if $c \geq 2 \sqrt{D_{0} f^{\prime}(0)}$ and they are stable with respect to some natural classes of perturbations, see e.g. [8, 28, 29, 42, 46]. For heterogeneous equations such as (1.1) in periodic media, standard traveling fronts do not exist in general and the notion of fronts is replaced by the more general one of pulsating fronts [45]. For (1.1), in the case of the existence (and uniqueness) of a periodic positive steady state $p(x)$ of $(2.2)$, a pulsating front connecting 0 and $p$ is a solution of the type $u(t, x)=\phi(x \cdot e-c t, x)$ with $c \neq 0, e$ is a unit vector $\left(e \in \mathbb{S}^{N-1}\right)$, and the function $\phi: \mathbb{R} \times \mathbb{R}^{N} \rightarrow \mathbb{R}$ satisfies

$$
\left\{\begin{array}{l}
\phi(-\infty, x)=p(x), \quad \phi(+\infty, x)=0 \text { uniformly in } x \in \mathbb{R}^{N}, \\
\phi(s, \cdot) \text { is } L \text {-periodic in } \mathbb{R}^{N}, \text { for all } s \in \mathbb{R} .
\end{array}\right.
$$

Notice that, if $u(t, x)=\phi(x \cdot e-c t, x)$ is a pulsating front, then, for every $x \in \mathbb{R}^{N}$, the function $t \mapsto u(t, x+c t e)$ is in general quasi-periodic.

The following result states the existence and uniqueness of the pulsating fronts.

Theorem $3.1[7,24]$ Under the assumptions of Theorem 2.1 with $\lambda[D, r]<0$, for every unit vector $e$ of $\mathbb{R}^{N}$, there exists a real number $c_{D, r, e}^{*}>0$ such that the pulsating fronts $u(t, x)=\phi_{c}(x \cdot e-c t, x)$ connecting 0 and $p$ for $(1.1)$ exist if and only if $c \geq c_{D, r, e}^{*}$. The minimal speed $c_{D, r, e}^{*}$ is characterized by the formula

$$
c_{D, r, e}^{*}=\min _{\lambda>0} \frac{-k_{D, r, e, \lambda}}{\lambda},
$$

where $k_{D, r, e, \lambda}$ denotes the principal eigenvalue of the operator

$$
L_{D, r, e, \lambda} \psi=-\nabla \cdot(D(x) \nabla \psi)+2 \lambda e D(x) \nabla \psi+\left[\lambda \nabla \cdot(D(x) e)-\lambda^{2} e D(x) e-r(x)\right] \psi
$$

with L-periodicity conditions. Furthermore, for each fixed speed $c \geq c_{D, r, e}^{*}$, the pulsating fronts $u(t, x)=\phi_{c}(x \cdot e-c t, x)$ are increasing in time and unique up to shifts in time.

Notice in particular that, if $D=D_{0}$ and $r=r_{0}$ are constant, then, by uniqueness of the principal eigenvalue, one has $k_{D, r, e, \lambda}=k_{D_{0}, r_{0}, e, \lambda}=-\lambda^{2} e D_{0} e-r_{0}$ and $c_{D, r, e}^{*}=2 \sqrt{\left(e D_{0} e\right) r_{0}}$. We also refer to $[2,5,20,27,31,32,47]$ for further existence, uniqueness and qualitative results, to $[34,37,38]$ for some results in space-time periodic media and $[2,5,10,15,19,26$, $43,48,49,50]$ for some existence and qualitative results with other types of nonlinearities or various boundary conditions in periodic domains.

In the following stability result, for every speed $c \geq c_{D, r, e}^{*}$, we denote $\lambda_{D, r, e, c}$ the smallest root of the equation $k_{D, r, e, \lambda}+c \lambda=0$ and $\psi_{D, r, e, c}$ a principal eigenfunction of the operator $L_{D, r, e, \lambda_{D, r, e, c}}$ under $L$-periodicity conditions. 
Theorem 3.2 [24] Under the assumptions of Theorem 3.1, for each fixed speed $c \geq c_{D, r, e}^{*}$, the pulsating fronts $\phi_{c}(x \cdot e-c t, x)$ are stable in the following sense: there exists $\varepsilon>0$ such that, if the initial condition $u_{0}$ of (1.1) satisfies $0 \leq u_{0} \leq p$ in $\mathbb{R}^{N}$,

$$
\liminf _{\sigma \rightarrow-\infty} \inf _{x \in \mathbb{R}^{N}, x \cdot e \leq \sigma}\left(u_{0}(x)-p(x)\right)>-\varepsilon
$$

and $u_{0}(x) \sim A e^{-\lambda_{D, r, e, c} x \cdot e} \psi_{D, r, e, c}(x)$ (resp. $\left.u_{0}(x) \sim A(x \cdot e) e^{-\lambda_{D, r, e, c} x \cdot e} \psi_{D, r, e, c}(x)\right)$ for some $A>0$ as $x \cdot e \rightarrow+\infty$ in the case $c>c_{D, r, e}^{*}$ (resp. in the case $c=c_{D, r, e}^{*}$ ), then there is a real number $\tau$ such that the solution $u$ of (1.1) with initial condition $u_{0}$ satisfies

$$
u(t, x)-\phi_{c}(x \cdot e-c t+\tau, x) \rightarrow 0 \text { as } t \rightarrow+\infty \text { uniformly in } x \in \mathbb{R}^{N} .
$$

Actually, this result is equivalent to the fact that, if the initial condition $u_{0}$ is sufficiently close to $p$ as $x \cdot e \rightarrow-\infty$ and has the same exponential decay as a pulsating front with speed $c \geq c_{D, r, e}^{*}$, then the solution $u$ of (1.1) will converge to this front as $t \rightarrow+\infty$ uniformly in space. For further stability results and attractivity of the fronts with minimal speeds, we refer to $[10,18,23]$.

\subsection{Speed of propagation: effect of the underlying medium}

In this section, we analyze the effect of the environment on the propagation speeds $c_{D, r, e}^{*}$, as defined in the previous section. These minimal speeds are of great importance from the point of view of the applications. Indeed, under the assumptions of Theorem 3.1, it is known [3, $17,47]$ that, if $u(t, x)$ solves (1.1) with a bounded compactly supported and nonzero initial condition $u_{0}$, then $u$ spreads with a speed $w_{D, r, e}^{*}>0$ in every direction $e \in \mathbb{S}^{N-1}$, in the sense that $u(t, x+c t e) \rightarrow p(x)$ locally uniformly in $x \in \mathbb{R}^{N}$ as $t \rightarrow+\infty$ for every $0 \leq c<w_{D, r, e}^{*}$, while $u(t, x+c t e) \rightarrow 0$ locally uniformly in $x \in \mathbb{R}^{N}$ as $t \rightarrow+\infty$ for every $c>w_{D, r, e}^{*}$, where $w_{D, r, e}^{*}$ is given by

$$
w_{D, r, e}^{*}=\min _{e^{\prime} \in S^{N-1}, e^{\prime} \cdot e>0} \frac{c_{D, r, e^{\prime}}^{*}}{e^{\prime} \cdot e} .
$$

In particular, in dimension 1 , the spreading speeds $w_{D, r, \pm 1}^{*}$ coincide with the minimal speeds $c_{D, r, \pm 1}^{*}$, which are equal since, for every $\lambda$, the operator $L_{D, r,-1, \lambda}$ is the adjoint of the operator $L_{D, r, 1, \lambda}$.

In the following statements, we focus on the dependence of the minimal speeds $c_{D, r, e}^{*}$ with respect to the diffusion and reaction rates, and similar statements can be obtained for the spreading speeds $w_{D, r, e}^{*}$. Let us first study the positive effect of the heterogeneities on the propagation speeds, as a consequence of Theorems 2.2 and 3.1.

Theorem 3.3 [7] Assume that the diffusion $D$ is the identity matrix I. Under the notations of Theorem 2.2, if $\bar{r}>0$, then $c_{I, r, e}^{*} \geq c_{I, \bar{r}, e}^{*}$ for every direction $e \in \mathbb{S}^{N-1}$. Furthermore, if $\bar{r} \geq 0$ and $r \not \equiv 0$, then the speeds $c_{I, B r, e}^{*}$ are increasing with respect to $B>0$, for every $e \in \mathbb{S}^{N-1}$.

Furthermore, the more aggregated the medium, the larger the speed, in the sense of the following result. 
Theorem 3.4 [35] Under the assumptions of the previous theorem, in dimension $N=1$, there holds $c_{I, r^{*}, \pm 1}^{*} \geq c_{I, r, \pm 1}^{*}$, where $r^{*}$ denotes the periodic symmetric decreasing Steiner rearrangement of the function $r$.

We point out that the same statement does not hold in general in higher dimensions $N \geq 2$, see [35]. In dimension $N=1$, another way to study the dependence of the propagation speeds on the fragmentation of the medium is to analyze the role of the spatial period $L=L_{1}$. We will in particular consider the homogenization limit $L \rightarrow 0^{+}$and the large scale limit $L \rightarrow+\infty$. To do so, we fix some functions $D$ and $f$ satisfying the general assumptions of Section 1 and being 1-periodic with respect to $x \in \mathbb{R}$. For $L>0$, we define

$$
D_{L}(x)=D\left(\frac{x}{L}\right) \text { and } f_{L}(x, u)=f\left(\frac{x}{L}, u\right) \text { for all }(x, u) \in \mathbb{R} \times[0,+\infty) .
$$

We also set $r(x)=\frac{\partial f}{\partial u}(x, 0)$ and $r_{L}(x)=r(x / L)=\frac{\partial f_{L}}{\partial u}(x, 0)$. When $L$ increases, the medium becomes somehow less fragmented and, following the general observations mentioned in Section 2.2 on the effect of fragmentation on species persistence, the pulsating fronts are expected to move faster in less fragmented media.

Theorem $3.5[12,21,22,35]$ In dimension $N=1$, if $\bar{r}>0$, then the function $L \mapsto c_{D_{L}, r_{L}, \pm 1}^{*}$ is nondecreasing and bounded on $(0,+\infty)$. Furthermore,

$$
c_{D_{L}, r_{L}, \pm 1}^{*} \rightarrow c_{0}^{*}:=2 \sqrt{D_{H} \bar{r}} \text { as } L \rightarrow 0^{+},
$$

where $D_{H}=1 /(\overline{1 / D})$ is the harmonic mean of the diffusion coefficient D. Lastly,

$$
c_{D_{L}, r_{L}, \pm 1}^{*} \rightarrow c_{\infty}^{*}:=\min _{\lambda \geq F(R), \lambda>0} \frac{F^{-1}(\lambda)}{\lambda} \text { as } L \rightarrow+\infty
$$

where $F^{-1}$ is the reciprocal of the function $F:[R, \infty) \rightarrow[F(R), \infty)$ defined by:

$$
F(s)=\int_{0}^{1} \sqrt{\frac{s-r(x)}{D(x)}} d x \text { for all } s \geq R:=\max _{x \in[0,1]} r(x) .
$$

These results show the different roles of the diffusion and growth rate coefficients in heterogeneous media and they provide some quantitative estimates of the influence of the spatial period, which can be measured by the ratio $c_{\infty}^{*} / c_{0}^{*} \geq 1$. For instance, if $D=D_{0}$ is constant and $r(x)=r^{+}>0$ on $(0,1 / 2)$ and $r(x)=0$ on $(1 / 2,1)$ (the function $r$ is not continuous in this case, but such a profile can be approximated by smooth functions, for which the limiting speeds $c_{0}^{*}$ and $c_{\infty}^{*}$ are close to the ones obtained in Theorem 3.5), then $c_{0}^{*}=\sqrt{2 D_{0} r_{+}}$, while $c_{\infty}^{*}=(8 / 9) \sqrt{3 D_{0} r_{+}}$, whence $c_{\infty}^{*}=(4 \sqrt{6} / 9) \times c_{0}^{*}$, independently of $D_{0}$ and $r_{+}$. Let us consider another example: if $r=r_{0}>0$ is constant, then $c_{0}^{*}=2 \sqrt{D_{H} r_{0}}$ and $c_{\infty}^{*}=2(\sqrt{D})_{H} \sqrt{r_{0}}$, where $(\sqrt{D})_{H}$ denotes the harmonic mean of $\sqrt{D}$. In this case, the ratio $c_{\infty}^{*} / c_{0}^{*}=(\sqrt{D})_{H} / \sqrt{D_{H}} \geq 1$ measures the relative increase of the propagation speeds between a homogenized medium and a slowly oscillating one. It can also be seen from Theorem 3.5 (see [22]) that, in some sense, the minimal speeds are much more sensitive to small 
perturbations (in the $L^{1}(0,1)$ sense) of the diffusion coefficients than to small perturbations of the growth rate. Another interesting consequence of Theorem 3.5 is the derivation of an equivalent of the propagation speed for large reaction terms: namely, under the assumptions and notations of Theorem 3.5, one has, for each fixed $L>0, c_{D_{L}, B r_{L}, \pm 1}^{*} \sim c_{0}^{*} \times \sqrt{B}$ as $B \rightarrow 0^{+}$ and $c_{D_{L}, B r_{L}, \pm 1}^{*} \sim c_{\infty}^{*} \times \sqrt{B}$ as $B \rightarrow+\infty$. We also point out that more general results hold in higher dimensions, see e.g. [11, 22, 35].

For further dependence results and optimal bounds for the propagation speeds in terms of the diffusion and growth rate coefficients in periodic media, we refer to $[5,11,30,36]$. Lastly, we mention that much work has been devoted to the study of the asymptotics of propagation speeds for reaction-diffusion equations with large advection terms in periodic media, see e.g. $[4,13,14,25,39,41,51,52]$.

\section{References}

[1] H. Berestycki, Le nombre de solutions de certains problèmes semi-linéaires elliptiques, J. Funct. Anal. 40 (1981), 1-29.

[2] H. Berestycki, F. Hamel, Front propagation in periodic excitable media, Comm. Pure Appl. Math. 55 (2002), 949-1032.

[3] H. Berestycki, F. Hamel, G. Nadin, Asymptotic spreading in heterogeneous diffusive media, J. Funct. Anal. 255 (2008), 2146-2189.

[4] H. Berestycki, F. Hamel, N. Nadirashvili, Elliptic eigenvalue problems with large drift and applications to nonlinear propagation phenomena, Comm. Math. Phys. 253 (2005), 451-480.

[5] H. Berestycki, F. Hamel, N. Nadirashvili, The speed of propagation for KPP type problems. I Periodic framework, J. Eur. Math. Soc. 7 (2005), 173-213.

[6] H. Berestycki, F. Hamel, L. Roques, Analysis of the periodically fragmented environment model : I - Species persistence, J. Math. Biology 51 (2005), 75-113.

[7] H. Berestycki, F. Hamel, L. Roques, Analysis of the periodically fragmented environment model : II - Biological invasions and pulsating travelling fronts, J. Math. Pures Appl. 84 (2005), 1101-1146.

[8] M. Bramson, Convergence of solutions of the Kolmogorov equation to travelling waves, Memoirs Amer. Math. Soc. 44, 1983.

[9] R.S. Cantrell, C. Cosner, Spatial Ecology via Reaction-Diffusion Equations, Series In Mathematical and Computational Biology, John Wiley and Sons, Chichester, Sussex UK, 2003.

[10] A. Ducrot, T. Giletti, H. Matano, Existence and convergence to a propagating terrace in onedimensional reaction-diffusion equations, Trans. Amer. Math. Soc. (2014), forthcoming.

[11] M. El Smaily, Pulsating travelling fronts: Asymptotics and homogenization regimes, Europ. J. Appl. Math. 19 (2008), 393-434.

[12] M. El Smaily, F. Hamel, L. Roques, Homogenization and influence of fragmentation in a biological invasion model, Disc. Cont. Dyn. Systems A 25 (2009), 321-342.

[13] M. El Smaily, S. Kirsch, The speed of propagation for KPP reaction-diffusion equations within large drift, Adv. Diff. Eq. 16 (2011), 361-400. 
[14] M. El Smaily, S. Kirsch, Front speed enhancement by incompressible flows in three or higher dimensions, Arch. Ration. Mech. Anal. (2014), forthcoming.

[15] J. Fang, X.-Q. Zhao, Bistable traveling waves for monotone semiflows with applications, J. Europ. Math. Soc., forthcoming.

[16] R.A. Fisher, The advance of advantageous genes, Ann. Eugenics 7 (1937), 335-369.

[17] M. Freidlin, J. Gärtner, On the propagation of concentration waves in periodic and random media, Sov. Math. Dokl. 20 (1979), 1282-1286.

[18] T. Giletti, Convergence to pulsating traveling waves with minimal speed in some KPP heterogeneous problems, Calc. Var. Part. Diff. Eq. (2014), forthcoming.

[19] J.-S. Guo, F. Hamel, Propagation and blocking in periodically hostile environments, Arch. Ration. Mech. Anal. 204 (2012), 945-975.

[20] F. Hamel, Qualitative properties of KPP and monostable fronts: monotonicity and exponential decay, J. Math. Pures Appl. 89 (2008), 355-399.

[21] F. Hamel, J. Fayard, L. Roques, Spreading speeds in slowly oscillating environments, Bull. Math. Biol. 72 (2010), 1166-1191.

[22] F. Hamel, G. Nadin, L. Roques, A viscosity solution method for the spreading speed formula in slowly varying media, Indiana Univ. Math. J. 60 (2011), 1229-1247.

[23] F. Hamel, J. Nolen, J.-M. Roquejoffre, L. Ryzhik, The logarithmic delay of KPP fronts in a periodic medium, preprint.

[24] F. Hamel, L. Roques, Uniqueness and stability properties of monostable pulsating fronts, J. Europ. Math. Soc. 13 (2011), 345-390.

[25] S. Heinze, Large convection limits for KPP fronts, preprint.

[26] J. Huang, W. Shen, Speeds of spread and propagation for KPP models in time almost and space periodic media, SIAM J. Appl. Dyn. Syst. 8 (2009), 790-821.

[27] W. Hudson, B. Zinner, Existence of travelling waves for reaction-diffusion equations of Fisher type in periodic media, In: Boundary Value Problems for Functional-Differential Equations, J. Henderson (ed.), World Scientific, 1995, 187-199.

[28] A.N. Kolmogorov, I.G. Petrovsky, N.S. Piskunov, Étude de l'équation de la diffusion avec croissance de la quantité de matière et son application à un problème biologique, Bull. Univ. Etat Moscou, Série Intern. A 1 (1937), 1-26.

[29] K.-S. Lau, On the nonlinear diffusion equation of Kolmogorov, Petrovsky, and Piscounov, J. Diff. Eq. 59 (1985), 44-70.

[30] X. Liang, X. Lin, H. Matano, A variational problem associated with the minimal speed of travelling waves for spatially periodic reaction-diffusion equations, Trans. Amer. Math. Soc. 362 (2010), 56055633.

[31] X. Liang, X.Q. Zhao, Asymptotic speeds of spread and traveling waves for monotone semiflows with applications, Comm. Pure Appl. Math. 60 (2007), 1-40.

[32] X. Liang, X.Q. Zhao, Spreading speeds and traveling waves for abstract monostable evolution systems, J. Funct. Anal. 259 (2010), 857-903. 
[33] J.D. Murray, Mathematical Biology, Springer-Verlag, 2003.

[34] G. Nadin, Travelling fronts in space-time periodic media, J. Math. Pures Appl. 92 (2009), 232-262.

[35] G. Nadin, The effect of Schwarz rearrangement on the periodic principal eigenvalue of a nonsymmetric operator, SIAM J. Math. Anal. 41 (2010), 2388-2406.

[36] G. Nadin, Some dependence results between the spreading speed and the coefficients of the space-time periodic Fisher-KPP equation, Europ. J. Appl. Math. 22 (2011), 169-185.

[37] J. Nolen, M. Rudd, J. Xin, Existence of KPP fronts in spatially-temporally periodic advection and variational principle for propagation speeds, Dyn. Part. Diff. Eq. 2 (2005), 1-24.

[38] J. Nolen, J. Xin, Existence of KPP type fronts in space-time periodic shear flows and a study of minimal speeds based on variational principle, Disc. Cont. Dyn. Syst. 13 (2005), 1217-1234.

[39] A. Novikov, L. Ryzhik, Boundary layers and KPP fronts in a cellular flow, Arch. Ration. Mech. Anal. 184 (2007), 23-48.

[40] L. Roques, F. Hamel, Mathematical analysis of the optimal habitat configurations for species persistence, Math. Biosciences 210 (2007), 34-59.

[41] L. Ryzhik, A. Zlatoš, KPP pulsating front speed-up by flows, Commun. Math. Sci. 5 (2007), 575-593.

[42] D.H. Sattinger, On the stability of waves of nonlinear parabolic systems, Adv. Math. 22 (1976), 312-355.

[43] W. Shen, Variational principle for spreading speeds and generalized propagating speeds in time almost periodic and space periodic KPP models, Trans. Amer. Math. Soc. 362 (2010), 5125-5168.

[44] N. Shigesada, K. Kawasaki, Biological Invasions: Theory and Practice, Oxford Series in Ecology and Evolution, Oxford: Oxford UP, 1997.

[45] N. Shigesada, K. Kawasaki, E. Teramoto, Traveling periodic waves in heterogeneous environments, Theor. Pop. Bio. 30 (1986), 143-160.

[46] K. Uchiyama, The behavior of solutions of some semilinear diffusion equation for large time, J. Math. Kyoto Univ. 18 (1978), 453-508.

[47] H.F. Weinberger, On spreading speeds and traveling waves for growth and migration in periodic habitat, J. Math. Biol. 45 (2002), 511-548.

[48] X. Xin, Existence and stability of travelling waves in periodic media governed by a bistable nonlinearity, J. Dyn. Diff. Eq. 3 (1991), 541-573.

[49] X. Xin, Existence of planar flame fronts in convective-diffusive periodic media, Arch. Ration. Mech. Anal. 121 (1992), 205-233.

[50] J.X. Xin, Analysis and modeling of front propagation in heterogeneous media, SIAM Review 42 (2000), 161-230.

[51] A. Zlatoš, Sharp asymptotics for KPP pulsating front speed-up and diffusion enhancement by flows, Arch. Ration. Mech. Anal. 195 (2010), 441-453.

[52] A. Zlatoš, Reaction-diffusion front speed enhancement by flows, Ann. Inst. H. Poincaré, Anal. Non Lin. 28 (2011), 711-726. 